\title{
FIXAÇÃO DAS FRATURAS DO PLATÔ TIBIAL COM PLACAS PELA VIA DE ACESSO POSTERIOR - RELATO PRELIMINAR DE 12 CASOS
}

\author{
TIBIAL PLATEAU FRACTURES FIXATION USING POSTERIOR APPROACHES - \\ PRELIMINARY RESULTS OF 12 CASES
}

\author{
André Wajnsztejn', Eduardo Fiorentino Alves de Araújo', Marcos Roberto Mellega'2, Luiz Fernando Cocco', \\ Wesley Max Ramos ${ }^{3}$, Daniel Balbachevsky ${ }^{3}$, Hélio Jorge Alvachian Fernandes ${ }^{4}$, Fernando Baldy dos Reis ${ }^{5}$
}

\section{RESUMO}

Objetivo: Apresentar os resultados preliminares com técnicas de abordagem posterior para fraturas do platô tibial, com traço de cisalhamento no plano sagital. Métodos: Doze pacientes com fraturas do platô tibial tratados cirurgicamente através de um acesso posterior direto foram incluídos no estudo. Foram revisados os prontuários, as radiografias e tomografias dos pacientes bem como as avaliações clínicas. Todos os casos foram acompanhados até o momento da consolidação óssea, definido como aquele em que o paciente apresentava sinais radiográficos compatíveis sendo capaz de apoiar carga total sobre o membro sem referir dor. Resultados: Entre julho de 2009 e abril de 2010, foram atendidos em nosso serviço 89 pacientes com fraturas da extremidade proximal da tíbia. Desses, 80 (89,9\%) foram tratados cirurgicamente. Doze pacientes $(13,6 \%)$ apresentavam uma fratura com um componente de cisalhamento posterior e, portanto, foram submetidos a abordagens posteriores para redução e fixação da fratura. Em três destes casos associou-se uma abordagem antero-lateral. A média de idades dos pacientes foi de 35 anos. O acompanhamento médio foi de 12 meses (entre 8 e 23 meses). As fraturas foram classificadas de acordo com o sistema AO/OTA: cinco como 41 B1, quatro como 41 B3, duas como $41 \mathrm{C} 1$ e uma como $41 \mathrm{C} 3$. Dentre as complicações, tivemos uma deiscência de sutura, tratada com curativos, e uma perda de redução que necessitou de uma reoperação. Em nenhum caso ocorreu lesão neurovascular, retarde de consolidação, pseudartrose ou instabilidade articular residual. Em 4 casos a redução foi considerada ruim ( $>2 \mathrm{~mm}$ de degrau articular), em 5 casos foi considerada imperfeita ( $<2 \mathrm{~mm}$ de degrau articular) e em 3 casos obtivemos uma redução anatômica. Conclusão: Os autores concluem que o uso da abordagem posterior deve ser considerada em casos de fraturas com onde haja componente de cisalhamento posterior do planalto da tíbia. Uma casuística ampliada é necessária para avaliar o real benefício desta abordagem.

Descritores: Fratura do Planalto Tibial; Via de Acesso; Tratamento

\section{ABSTRACT}

Objective: To describe our preliminary results of posterior shearing tibia plateau fractures treated by a direct dorsal approach and plate fixation. Methods: A consecutive series of twelve patients with tibia plateau fractures treated by direct posterior approach was selected from our database. Conventional radiographies, computed tomography scans and medical records were reviewed. All cases were followed to union, as defined by painless weight bearing and radiographic healing. Results: Between July 2009 and April 2010, our trauma service received 89 tibia plateau fractures and treated 80 (89,9\%) operatively. Twelve patients $(13,5 \%)$ sustained posterior shearing tibia plateau fractures. All fractures were treated through the posterior approach, although 3 required association with an anterolateral approach as well. The mean age of patients was 35 years and mean follow-up was 12 (range 8-23) months. The fractures were classified according to AO/OTA: five $41 \mathrm{B1}$, four $41 \mathrm{B3}$, two $41 \mathrm{Cl}$ and one 41 C3. There was one wound dehiscence, managed with local wound care, and one loss of reduction treated by reoperation. No patient sustained neurovascular injury, nonunion, malunions or knee instability. In four cases the reduction was rated as poor ( $>2 \mathrm{~mm}$ step off), in five cases reduction was rated as imperfect $(<2 \mathrm{~mm}$ step off) and in three cases the reduction was rated as anatomic (absolutely no step-off). Conclusion: Authors conclude that posterior approaches should be considered when tibial plateau fractures result in posterior displaced fragments. A larger sample is needed to get definitive conclusions.

Keywords: Tibial Plateau Fracture; Surgical Approach; Treatment

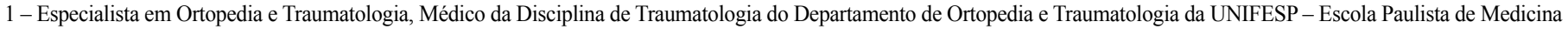

2 - Residente do $2^{\circ}$ ano do Departamento de Ortopedia e Traumatologia da UNIFESP - Escola Paulista de Medicina

3 - Mestre em Ciências do Departamento de Ortopedia e Traumatologia da UNIFESP - Escola Paulista de Medicina

4 - Doutor em Ciências do Departamento de Ortopedia e Traumatologia da UNIFESP - Escola Paulista de Medicina

5 - Livre Docente do Departamento de Ortopedia e Traumatologia da UNIFESP - Escola Paulista de Medicina

Estudo desenvolvido no Departamento de Ortopedia e Traumatologia da Universidade Federal de São Paulo - UNIFESP - Escola Paulista de Medicina

Correspondência: R. Borges Lagoa, 783, $5^{\circ}$ andar, Vila Clementino, São Paulo-SP. Endereço eletrônico: aw6868@hotmail.com

Trabalho recebido para publicação: 15/03/11, aceito para publicação: 15/04/11 


\section{INTRODUÇÃO}

As fraturas do platô tibial são relativamente raras, representando aproximadamente $1,2 \%$ de todas as fraturas, com frequência semelhante a das fraturas do calcâneo e da diáfise do úmero ${ }^{(1)}$.

A direção e magnitude do trauma, bem como a posição do joelho na hora do impacto determinam o padrão da fratura, sua localização e grau de desvio. Geralmente as fraturas com um componente cisalhante posterior costumam decorrer de um trauma com compressão axial com o joelho fletido ou semi-fletido.

Nesses casos que o traço da fratura ocorre no plano coronal, esse fragmento tende a cisalhar em direção posterior, e a fixação deste fragmento é um desafio ao ortopedista, não existindo consenso quanto ao melhor método de estabilizá-lo. Pode ser fixado por via anterior através de parafusos de tração da frente para trás, ou por via posterior e colocação de placas de apoio, teoricamente mais eficientes do ponto de vista biomecânico.

Avanços significativos foram feitos no modo de diagnosticar e tratar essas lesões. A contribuição da medicina diagnóstica, através da tomografia computadorizada e da ressonância magnética, o aperfeiçoamento dos implantes ortopédicos e novas publicações mudaram a conduta nessas lesões.

Diversas classificações foram propostas para estas fraturas. Podemos destacar a classificação de Hohl e modificada por Moore e Hohl (Figura 1). Essa classificação distingue 5 tipos de fratura e 5 tipos de fraturaluxação. Dentre os tipos de fratura-luxação, o tipo I representa uma fratura tipo cisalhamento no plano coronal, justamente a qual pode ser tratada através de um acesso posterior direto ${ }^{(2)}$.

De acordo com a classificação de Schatzker ${ }^{(3)}$, todos os tipos podem ocorrer com um cisalhamento posterior. Apesar de ser amplamente difundida, ela não faz uma referência direta as fraturas com um traço no plano coronal. Os três primeiros grupos (I, II e III) são fraturas puras, enquanto que os grupos IV, V e VI são fraturasluxações ${ }^{(4)}$.

A classificação da $\mathrm{AO} / \mathrm{OTA}^{(5)}$ apresenta o tipo parcialmente articular 41 B. Seus subtipos 41 B1 representa uma fratura tipo cisalhamento, no subtipo 41 B2 representa uma fratura tipo depressão e $41 \mathrm{~B} 3$ representa uma lesão combinada de cisalhamento e depressão. Acrescentando-se o número 1 (41 B1.1) temos uma lesão tipo cisalhamento no planalto lateral, acrescentando-se o número 2 uma lesão no planalto medial e o número 3 uma fratura oblíqua que envolve as eminências intercondileanas e um dos planaltos. O número 4 seguinte (p.ex. $41 \mathrm{~B} 1.14$ ) representa a fratura frontal posterior.

Existe um esquema de 3 colunas proposto por Luo et $a l^{(6)}$ que apresenta a vantagem de indicar a via que devemos utilizar em cada fratura (Figura 2). Nas fraturas que apresentam um componente cisalhante posterior, é proposto um acesso cirúrgico posterior direto.

O objetivo deste estudo é apresentar os resultados preliminares de abordagens posteriores para o tratamento de fraturas do planalto tibial com cisalhamento posterior de fragmentos articulares.

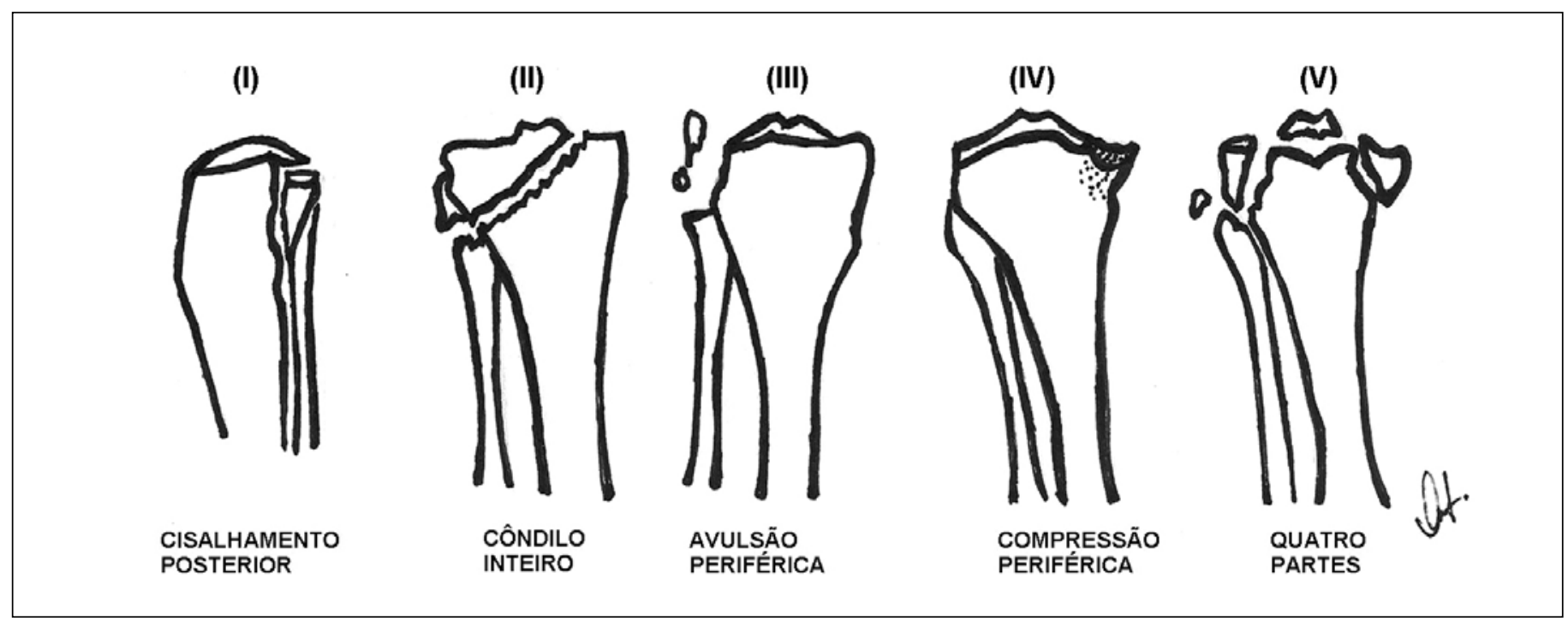

Figura 1 - Classificação de Hohl e Moore. Detalhe do tipo I que corresponde às fraturas no plano coronal que cisalham em direção posterior. O tipo II corresponde a uma fratura de todo o côndilo, tipo III uma avulsão da borda, tipo IV uma fratura tipo compressão em sua borda e a tipo $\mathrm{V}$ uma fratura em 4 partes do platô tibial. 


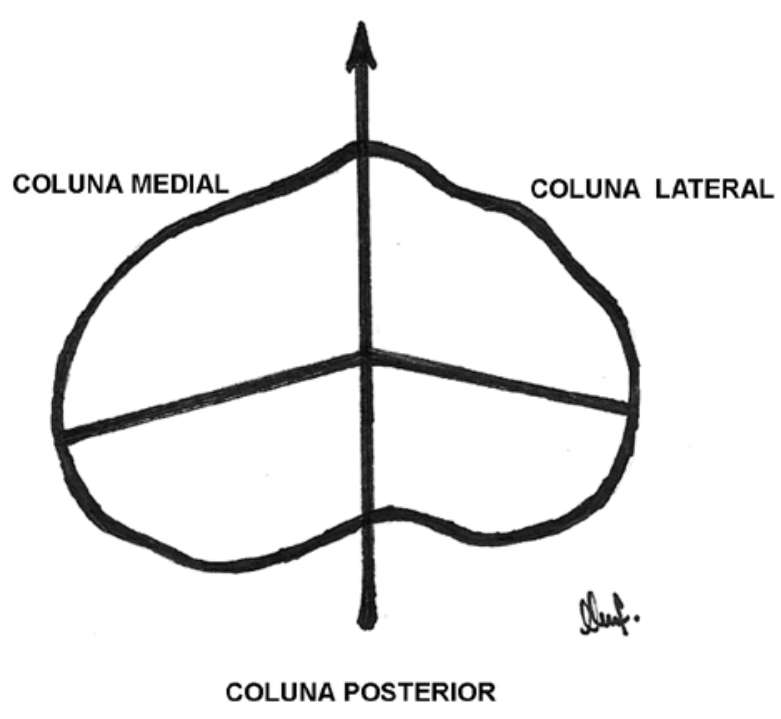

Figura 2 - Ilustração demonstrando a divisão em 3 colunas do platô tibial, através de uma imagem axial, vista superior. Coluna lateral, medial e posterior.

\section{MÉTODOS}

Este é um estudo retrospectivo de série de casos.

Doze pacientes com fraturas do platô tibial no plano coronal (tipo I de Hohl e Moore), tratados cirurgicamente através de acesso posterior, foram incluídos em nossa casuistica.

Em todos os casos, os pacientes foram posicionados em decúbito ventral e foi instalado um garrote pneumático, para ser insuflado caso necessário.

Nos pacientes que apresentavam apenas uma fratura na porção póstero-lateral, utilizamos a incisão cutânea preconizada por Carlson ${ }^{(7)}$. Carlson preconizou acessos separados para fraturas bicondilares. Utilizamos somente sua porção lateral. Após a dissecção do subcutâneo buscamos o nervo fibular no sentido de proximal para distal. O nervo deve ser afastado lateralmente junto com o tendão do bíceps femoral. A cabeça lateral do gastrocnêmio deve ser afastada medialmente juntamente com a artéria poplítea.

Nos pacientes que apresentavam apenas uma fratura na porção póstero-medial, utilizamos a via de Lobenhoffer modificada $^{(8)}$. Essa via foi originalmente descrita em alemão e foi publicada em inglês pela primeira vez em $2007^{(9)}$. É feita uma incisão cutânea longitudinal na região entre a cabeça medial do gastrocnêmio e os tendões flexores do joelho. Pode ser feita uma progressão transversal na prega flexora do joelho. Faz-se uma dissecção romba entre o semimembranoso e a cabeça medial do gastrocnêmio. Podemos afastar gentilmente a cabeça medial do gastrocnêmio lateralmente e os tendões flexores lateralmente e acessar a cápsula póstero-medial. Proximalmente é possível visualizar o ligamento poplíteo oblíquo e a origem do músculo solear. Alguns autores preconizam que pode ser reparado o tendão do gastrocnêmio medial ${ }^{(10)}$, porém em nossa série isso não foi necessário em nenhum caso.

Nos pacientes que apresentavam fraturas com componente cisalhante póstero-lateral e póstero-medial, utilizamos a via cutânea em "S", através da prega de flexão do joelho preconizada por Burks e Schaffer ${ }^{(12)}$. Sua parte superior deve permanecer na parte lateral e a parte inferior na medial. Os planos mais profundos são iguais aos já descritos nos acessos póstero-lateral e póstero-medial.

Para a fixação da fratura utilizamos, placas do tipo DCP, placas de reconstrução ou placas em " $T$ " de $3,5 \mathrm{~mm}$ isoladas ou associadas a parafusos de tração com arruela.

Foram revisados os prontuários, as radiografias e tomografias dos pacientes bem como as avaliações clínicas. Todos os casos foram acompanhados até a consolidação, definida através de carga total indolor e sinais radiográficos compatíveis.

\section{RESULTADOS}

Entre julho de 2009 e abril de 2010, foram atendidos em nosso serviço 89 pacientes com fraturas da extremidade proximal da tíbia. Desses, 80 (89,9\%) foram tratados cirurgicamente.

Doze pacientes $(13,6 \%)$ apresentavam uma fratura com um componente cisalhante posterior. Nove pacientes foram vítimas de acidentes com motocicletas, 2 pacientes vítimas de acidentes automobilísticos e 1 vítima de queda. Estes pacientes foram tratados por um acesso posterior direto, 3 casos necessitaram de um acesso ântero-lateral associado. A média das idades dos pacientes foi de 35 anos. O acompanhamento médio foi de 12 meses (entre 8 e 23 meses).

\section{PADRÕES DE FRATURAS}

As fraturas foram classificadas de acordo com Hohl e Moore (todas tipo I) e pelo sistema AO/OTA: cinco como $41 \mathrm{~B} 1$, quatro como $41 \mathrm{~B} 3$, duas como $41 \mathrm{C} 1 \mathrm{e}$ uma como 41 C3 (Gráfico 1). 


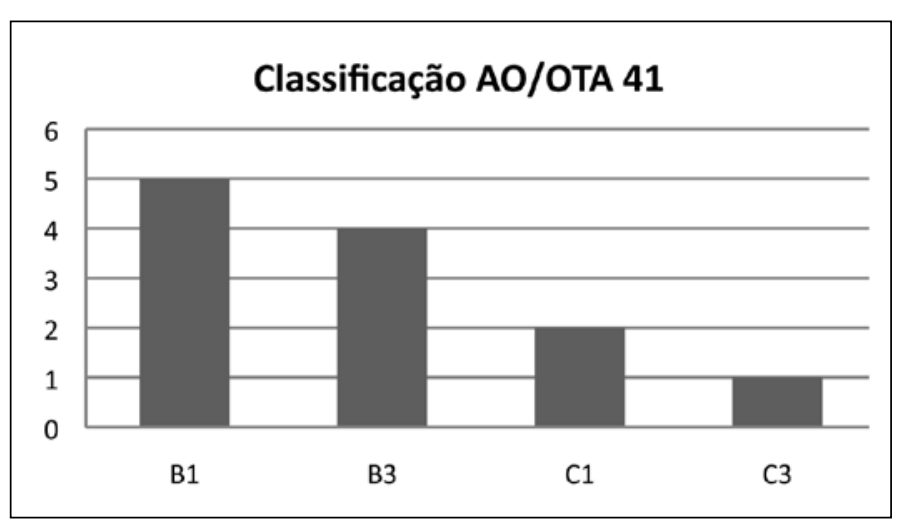

Figura 1 - Distribuição das fraturas de acordo com a classificação AO/OTA.

\section{RESULTADOS CLÍNICOS}

Dentre as complicações, tivemos uma deiscência de sutura, tratada com curativos. Nenhum caso de lesão neurológica ou vascular. Nenhum retardo de consolidação ou pseudartrose. Em um caso tivemos uma perda de redução que necessitou de uma reoperação.

$\mathrm{O}$ arco de movimento variou entre 0 e 15 graus (media de -7,5) de extensão e 95 e 140 graus (media de 117) de flexão. Nenhum paciente apresentou instabilidade no joelho (Tabela 1).

Tabela 1 - Resultados clínicos.

\begin{tabular}{c|c|c|c|c|c|c}
\hline Paciente & Idade & Sexo & Mecanismo & Redução & $\begin{array}{c}\text { Arco de } \\
\text { movimento }\end{array}$ & Complicações \\
\hline 1 & 25 & M & Moto & Anatômica & 0 a 130 & \\
\hline 2 & 53 & M & Queda & Imperfeita & 5 a 100 & $\begin{array}{c}\text { Deiscência de } \\
\text { sutura }\end{array}$ \\
\hline 3 & 40 & M & Auto & Anatômica & 0 a 140 & \\
\hline 4 & 19 & M & Moto & Imperfeita & 10 a 120 & \\
\hline 5 & 17 & M & Moto & Imperfeita & 10 a 120 & \\
\hline 6 & 50 & M & Moto & Ruim & 10 a 130 & \\
\hline 7 & 33 & M & Moto & Imperfeita & 10 a 110 & \\
\hline 8 & 44 & M & Moto & Ruim & 5 a 95 & \\
\hline 9 & 23 & M & Moto & Imperfeita & 15 a 110 & Perda de \\
redução
\end{tabular}

\section{QUALIDADE DA REDUÇÃO ARTICULAR}

Em 4 casos a redução foi considerada ruim $(>2 \mathrm{~mm}$ de degrau articular), em 5 casos foi considerada imperfeita ( $<2 \mathrm{~mm}$ de degrau articular) e em 3 casos obtivemos uma redução anatômica (Figura 2).

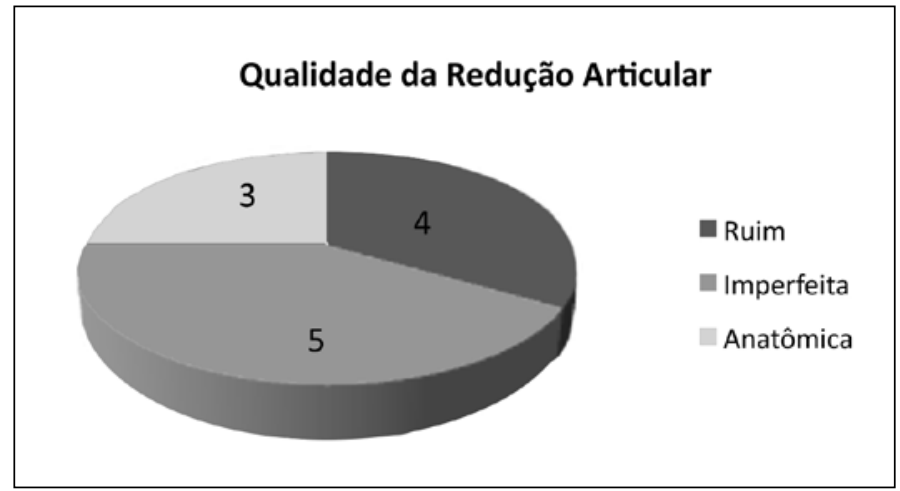

Figura 2 - Distribuição da qualidade de redução das fraturas através do acesso posterior.

\section{DISCUSSÃO}

A maioria das descrições das fraturas do planalto tibial são relacionadas as suas características no radiografia em ântero-posterior. Pouca atenção é dada ao desvio no plano sagital. A literatura é pobre em relatos de fraturas com um componente cisalhante posterior. De Boeck e Opdecam fizeram um relato com apenas 7 pacientes tratados com um acesso posterior ${ }^{(13)}$ enquanto que Bhattacharyya et $a l^{(14)}$ descreveram os resultados de 13 pacientes tratados por um acesso posterior direto. Recomendamos que sempre na suspeita de fraturas no plano coronal seja realizada tomografia computadorizada, para a melhor avaliação e entendimento da lesão.

As vias de acesso posterior ao joelho são usadas há muitos anos para o tratamento de lesões vasculares, cistos poplíteos, drenagem de abcessos, entre outros ${ }^{(15)}$. Acreditamos que as fraturas com cisalhamento posterior do platô tibial devam ser tratadas por um acesso posterior direto, pois oferece uma exposição ampla aos côndilos femorais, ao platô tibial e das partes moles da fossa poplítea, incluindo a artéria, veia e nervo tibial, nervo fibular comum e várias origens e inserções tendíneas. E com o devido afastamento e proteção desta estruturas neurovasculares, podemos ter acesso seguro à toda a metáfise proximal da tíbia, para redução e fixação das fraturas do platô tibial.

Diversas vias de acesso foram descritas para acessar essas fraturas diretamente, demonstrando o interesse de se fixar as fraturas de maneira mais estável. Assim como Fakler et al acreditamos que esta fixação mais estável e resistente é justificada pela magnitude das forças de carga que são impostas nesta região, pela ação dos côndilos femorais. Métodos de fixação menos resistentes, como parafusos de tração apenas, colocados de anterior para posterior podem ter mais falhas de fixação. 
É importante lembrar que uma das limitações da via ao côndilo lateral, é que a exposição póstero-lateral é limitada distalmente pela trifurcação dos vasos através da membrana interóssea. Isso ocorre aproximadamente a $5 \mathrm{~cm}$ da linha articular, por isso não recomendamos o uso dessa via em fraturas que tenham um cisalhamento que se estenda a mais de $4 \mathrm{~cm}$ da linha articular.

\section{CONCLUSÃO}

Os autores concluem que vias de acesso posteriores ao joelho devam ser consideradas nos casos em que o cisalhamento da fratura ocorra no plano frontal levando ao cisalhamento posterior de fragmentos articulares. Acreditam ainda que uma casuística maior de casos com critérios de inclusão mais claros deva ser constituída para determinar o real valor destas vias de acesso.

\section{REFERÊNCIAS}

1. Court-Brown CM, Caesar B. Epidemiology of adult fractures: A review. Injury 2006 37(8):691-7.

2. Hohl M, Moore TM: Articular fractures of the proximal tibia. In: Evarts CM, ed: Surgery of the musculoskeletal system, 2nd ed, New York: Churchill Livingstone, 1990.

3. Schatzker J, Tile M. The rational of operative fracture care. 3rd ed. SpringerVerlag, 2005.

4. Kfuri M Jr, Fagagnolo F, Bitar R C, Freitas R L, Salim R, Paccola C A J. Fraturas do planalto tibial. Rev Bras Ortop 2009; 44(5): 468-474.

5. Orthopaedic Trauma Association Committee for Coding and Classification (1996) Fracture and dislocation compendium. J Orthop Trauma 1996;10 Supp 1:v-ix, 1-154.

6. Luo C-F, Sun H, Zhang B, Zeng B-F. Three-Column fixation for complex tibial plateau fractures. J Orthop Trauma 2010 Nov;24(11):683-92.

7. Carlson DA. Bicondylar fracture of the posterior aspect of the tibial plateau-a case report and a modified approach. J Bone Joint Surg Am. 1998 Jul;80(7):1049-52.

8. Galla M, Lobenhoffer P. The direct, dorsal approach to the treatment of unstable tibial posteromedial fracture-dislocations (German). Unfallchirurg. 2003 Mar;106(3):241-7.
9. Fakler JKM, Ryzewicz M, Hartshorn C, Morgan SJ, Stahel PF, Smith W, Optimizing the Management of Moore Type I Postero-Medial Split Fracture Dislocations of the Tibial Head: Description of the Lobenhoffer Approach. J Orthop Trauma 2007 May;21(5):330-6.

10. Alpert JM, McCarty LP, Bach BR Jr. The direct posterior approach to the knee - Surgical and anatomic approach. J Knee Surg. 2008 Jan;21(1):44-9.

11. Bermúdez CA, Ziran BH, Barrette-Grischow MK. Elements of Complex Tibial Plateau Fractures: Description . J Trauma. 2008 Nov;65(5):1162-7.

12. Burks RT, Schaffer JJ. A simplified approach to the tibial attachment of the posterior cruciate ligament. Clin Orthop Relat Res. 1990 May;(254):216-9.

13. De Boeck $H$, Opdecam P. Posteromedial tibial plateau fractures: operative treatment by posterior approach. Clin Orthop Relat Res. 1995 Nov;(320):125-8.

14. Bhattacharyya T, McCarty LP 3rd, Harris MB, Morrison SM, Wixted JJ, Vrahas MS, Smith RM. The posterior shearing tibial plateau - treatment and results via a posterior approach. J Orthop Trauma. 2005 May-Jun;19(5):305-10.

15. Abbot L C, Carpenter W F. Surgical approaches to the knee joint. J Bone Joint Surg Am. 1945; 27: 277-310.

16. Lobenhoffer P, Gerich T, Bertram T, Lattermann C, Pohlemann T, Tscheme H. Particular posteromedial and posterolateral approaches for the treatment of tibial head fractures. Unfallchirurg 1997 Dec;100(12):957-67. 